\title{
Impact of the decay width in Breit-Wigner formula on Maxwellian-averaged cross section for neutron capture on ${ }^{16} \mathrm{O}$
}

\author{
Si-Zhe Xu and Shi-Sheng Zhang \\ ${ }^{1}$ School of Physics, and International Research Center for Big-Bang Cosmology and Element Genesis, \\ Beihang University, Beijing 100191, P. R. China
}

\begin{abstract}
Neutron capture on ${ }^{16} \mathrm{O}$ may serve as a crucial neutron poison reaction in weak s-process occurred in massive stars. In our previous study [Zhang, S. S., Xu, S. Z., He, M. et al. Eur. Phys. J. A 57, 114 (2021)], we found that the contribution from low-lying resonances to Maxwellian-averaged cross section (MACS) progressively increases as the energy goes beyond $70 \mathrm{keV}$. In BreitWigner formula for resonant cross section, the decay width is a decisive quantity. In this paper, we discuss the impact of three kinds of decay widths, i.e. a constant width and two energy-dependent widths, on reaction cross sections and MACSs. The penetration factor adopts semi-classical WKB approximation and the asymptotic solution, respectively. We clarify that energy-dependent width are necessary for a reasonable behavior of resonance cross section around resonance peak and low-energy region far from the peak. The difference of two energy-dependent widths decreases from 3.7 to 1.5 with the energy increasing from $0.01 \mathrm{keV}$ to $1000 \mathrm{keV}$. It results in similar behavior of resonance cross sections, but a slight difference by $1 \%$ for MACSs when $\mathrm{E}>50 \mathrm{keV}$.
\end{abstract}

\section{Introduction}

Light nuclei play an important role in the studies of neutron capture nucleosynthesis. Some of them may absorb a large amount of neutrons, as so called "neutron poisons", which have an important impact on the synthesis of heavy elements. ${ }^{16} \mathrm{O}$ may be one of the candidates due to its high abundance and large reaction rate of neutron capture process. It has been shown that the difference of the ${ }^{16} \mathrm{O}(n, \gamma){ }^{17} \mathrm{O}$ rates apparently influence the weak $s$-process in massive stars [1], which trigger us to study the properties of ${ }^{16} \mathrm{O}(n, \gamma){ }^{17} \mathrm{O}$, especially for its cross section.

The cross section of neutron capture on ${ }^{16} \mathrm{O}$ mainly consists of three parts: direct capture, resonance, and the interference between the direct capture and the resonance. We have estimated these terms separately and found that the contribution from low-lying resonance to Maxwellian-averaged cross section (MACS) progressively increases as the energy goes beyond $70 \mathrm{keV}$, in which the resonant cross section takes the form of Breit-Wigner formula with an energy-dependent decay width $[2,3]$.

Usually, the decay width of a resonant state is defined as a constant in textbooks and some references $[4,5]$. Except for a constant width, an energy-dependent width is also utilized in some works [6-8], including our former attempts [2,3]. There are two ways commonly 
used to obtain an energy-dependent width. One way is to calculate the penetration factor by using semi-classical WKB approximation, then decay width as a function of energy can be evaluated. The other way is to express penetration factor by the asymptotic solution (AS) of Bessel function, which is adopted in our former work [3]. Here, we aim at clarifying the effectiveness and rationality among these three kinds of decay widths, and showing their impacts on resonant cross sections and MACS.

This paper is made up of three sections. In section 2, we simply review the theoretical framework to achieve penetration factors in two ways, i.e. WKB approximation and asymptotic solution. In section 3, we show the distinct impact of constant and energy-dependent decay widths on resonant cross sections and MACSs. Finally, we draw a simple summary in section 4 .

\section{Theoretical framework}

\subsection{Maxwellian-averaged cross section}

As key inputs of nucleosynthesis simulations, the reaction rates $N_{A}\langle\sigma v\rangle$ is equivalent to MACS $\left\langle\sigma>_{k_{B} T}\right.$ except for a factor, i.e., $\left.N_{A}<\sigma v\right\rangle=N_{A} v_{k_{B} T}<\sigma>_{k_{B} T}$, where $N_{A}$ is the Avogadro number, and $v_{k_{B} T}$ is the thermal velocity. The expression of MACS reads,

$$
<\sigma>_{k_{B} T}=\frac{2}{\sqrt{\pi}} \frac{1}{\left(k_{B} T\right)^{2}} \int_{0}^{\infty} \sigma(E) E \exp \left(-\frac{E}{k_{B} T}\right) d E
$$

which is the convolution of the reaction cross section $\sigma(E)$, the incident energy $E$, and exponential term with Boltzmann constant $k_{B}$ and temperature $T$.

\subsection{Breit-Wigner formula for resonant cross section}

The reaction cross section mainly consists of three parts: direct capture, resonance, and the interference between the direct capture and the resonance. The Breit-Wigner formula is commonly utilized for the estimation of the resonant cross section, written as

$$
\sigma_{R}(E)=\frac{\pi \hbar^{2}}{2 \mu E} \frac{2 J+1}{\left(2 J_{a}+1\right)\left(2 J_{X}+1\right)} \frac{\Gamma_{a} \Gamma_{b}}{\left(E-E_{r}\right)^{2}+(\Gamma / 2)^{2}},
$$

in which $\mu$ is the reduced mass, defined as $\mu=m_{a} m_{X} /\left(m_{a}+m_{X}\right), E_{r}$ is the resonance energy, and $E$ is the incident energy of the projectile; $J, J_{a}$ and $J_{X}$ refer to total angular momentums of the compound nucleus, the projectile $a$, and the target $X$, respectively; $\Gamma_{a}$ and $\Gamma_{b}$ are the decay widths of incoming particle $a$ and outgoing particle $b$, and total width $\Gamma$ is the summation of them. As shown in Breit-Wigner formula, the decay width of incoming particle $a$ is one of important resonance parameters, but lacks of detailed description in the textbooks. Usually, one regards decay width as a constant in the calculations. We will show the difference aroused by constant and energy-dependence widths in the following.

\subsection{The decay width}

The decay width usually increases with incident particle energy $E$ as the barrier decreases. An energy-dependence width takes the form as shown in Ref. [9]

$$
\Gamma_{l}=\frac{3 \hbar}{R} \sqrt{\frac{2 E}{\mu}} P_{l} \theta_{l}^{2}
$$


where $P_{l}$ is the penetration factor, and $\theta_{l}^{2}$ is the dimensionless reduced width, defined as $\theta_{l}^{2}=\chi_{l}^{*}(R) \chi_{l}(R) R / 3$. In this definition, $\Gamma_{l}$ is the function of the particle energy $E$, proportional to the penetration factor $P_{l}$ and $\sqrt{E}$.

\subsection{Penetration factor}

Penetration factor $P_{l}$ is defined as the ratio of the probabilities of particles appearing at infinity to those appearing at $R$, which can be expressed by the radial wave function. For $r>R$, The solution is a linear combination of regular Coulomb wave function $F_{l}(r)$ and irregular Coulomb wave function $G_{l}(r)$. Therefore, it reads

$$
P_{l}=\frac{\chi_{l}^{*}(\infty) \chi_{l}(\infty)}{\chi_{l}^{*}(R) \chi_{l}(R)}=\frac{1}{F_{l}^{2}(R)+G_{l}^{2}(R)} .
$$

\subsubsection{The asymptotic solution (AS)}

Without Coulomb interaction, the radial wave function can be simplified as a linear combination of spherical Bessel function $j_{l}(r)$ and Neumann function $n_{l}(r)$, i.e. so called the asymptotic solution (AS). Hence, the penetration factor can be rewritten as:

$$
P_{l}=\frac{1}{\rho^{2}\left(j_{l}^{2}(R)+n_{l}^{2}(R)\right)}
$$

where $\rho=k R$ with wave number $k$ and matter radius of the nucleus $R$. In Table 1 , we list the penetration factors $P_{l}$ and level shift $S_{l}$ for different reaction channels with angular momentum $l=0,1,2, \ldots$. Here, we mainly focus on the low-lying $2 p_{3 / 2}$ resonant state, in which $l=1$.

Table 1. Penetration factors $P_{l}$ and level shift $S_{l}$ for different reaction channels without Coulomb interaction [10].

\begin{tabular}{ccc}
\hline \hline$l$ & $P_{l}$ & $S_{l}$ \\
\hline 0 & 1 & 0 \\
1 & $\rho^{2} /\left(1+\rho^{2}\right)$ & $1 /\left(1+\rho^{2}\right)$ \\
2 & $\rho^{4} /\left(9+3 \rho^{2}+\rho^{4}\right)$ & $-\left(18+3 \rho^{2}\right) /\left(9+3 \rho^{2}+\rho^{4}\right)$ \\
$l$ & $\frac{\rho^{2} P_{l-1}}{\left(l-S_{l-1}\right)^{2}+\rho^{2} P_{l-1}^{2}}$ & $\frac{\rho^{2}\left(l-S_{l-1}\right)}{\left(l-S_{l-1}\right)^{2}+\rho^{2} P_{l-1}^{2}}-l$ \\
\hline \hline
\end{tabular}

\subsubsection{The WKB approximation}

The WKB approximation is commonly used for barrier tunneling penetration problems, which is the approximated solution of a differential equation like $y^{\prime \prime}=-f(x) y$. Here, we use $f(r)=2 \mu\left[E-V_{l}(r)\right] / \hbar$, so that the penetration factor follows [9]:

$$
P_{l}(E)=\frac{\chi_{l}^{*}(\infty) \chi_{l}(\infty)}{\chi_{l}^{*}(R) \chi_{l}(R)}=\left[\frac{V_{l}(R)}{E}-1\right]^{1 / 2} \exp \left[-\frac{2 \sqrt{2 \mu}}{\hbar} \int_{R}^{R_{0}} \sqrt{V_{l}(r)-E} d r\right],
$$

where $R$ denotes the nuclear matter radius, and $R_{0}$ is the classical turning point satisfied with $V_{l}\left(R_{0}\right)=E$. Since there is no Coulomb interaction for neutron capture process, it can be 
reduced to

$$
\int_{R}^{R_{0}} \sqrt{V_{l}(r)-E} d r=R\left[\sqrt{E_{l}} \ln \left(\sqrt{\frac{E_{l}}{E}}+\sqrt{\frac{E_{l}}{E}-1}\right)-\sqrt{E_{l}-E}\right] .
$$

Later on, we will discuss the impact of penetration factors on the decay widths, the impact of the energy-dependent widths on the resonant cross sections and MACSs.

\section{Results and discussions}

\subsection{Decay widths}

In last section, we introduce two ways to obtain penetration factors $P_{l}$, i.e. the AS and WKB approximation. Given the penetration factors, we can calculate the decay widths with Eq. 3. The dimensionless reduced width $\theta_{l}^{2}$ is also required. With measured resonance energy $E_{R}$ and width $\Gamma_{n}\left(E_{R}\right)$ from experiments, $\theta_{l}^{2}$ is easy to be determined by the Eq. 3 . For the case of ${ }^{16} \mathrm{O}(n, \gamma){ }^{17} \mathrm{O}$, the decay width $\Gamma_{n}=40 \mathrm{keV}$ and the resonant energy $E_{R}=411 \mathrm{keV}$, are lately measured [11]. Then, $\theta_{l}^{2}$ can be fixed to $5.128 \times 10^{-2}$ with $P_{l}^{A S}$ in Eq. 3. The matter radius of ${ }^{16} \mathrm{O}$ takes the value of $2.6 \mathrm{fm}$, and the angular momentum $l=1$ corresponding to p-orbital valence neutron.

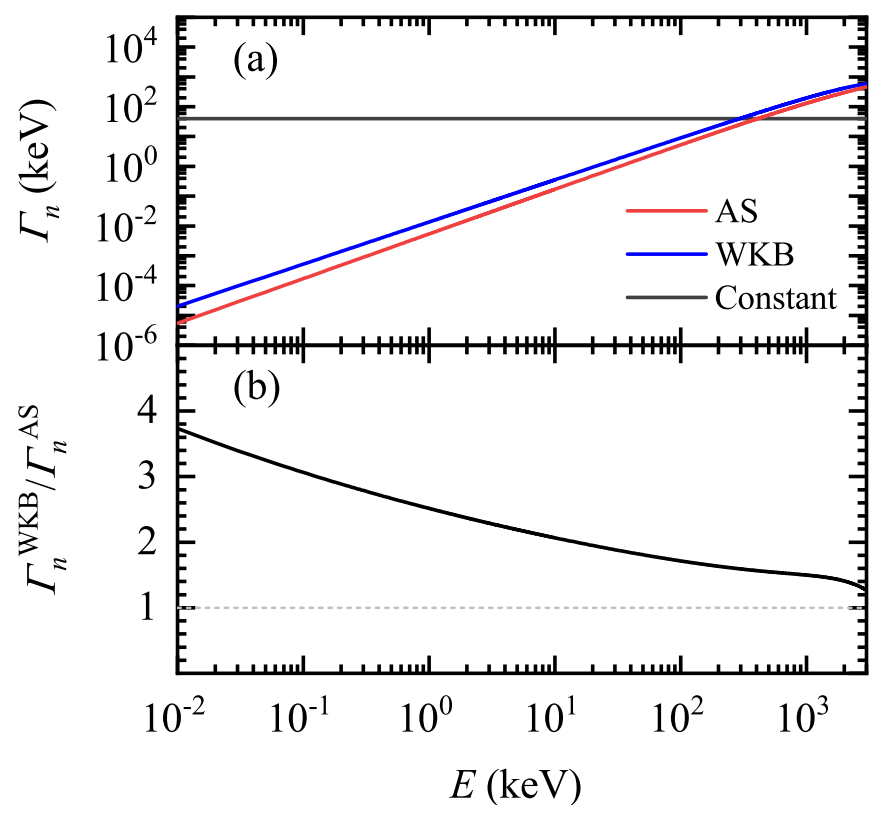

Figure 1. (a) A constant decay width (black line), and two decay widths $\Gamma_{n}(E)$ as a function of energy $E$, based on the penetration factors from the asymptotic solution (red line) and the WKB approximation (blue line), for ${ }^{16} \mathrm{O}(n, \gamma){ }^{17} \mathrm{O}$, respectively. (b) ratio of $\Gamma_{n}^{W K B}$ to $\Gamma_{n}^{A S}$ as a function of energy $E$.

We plot the decay widths $\Gamma_{n}(E)$ as a function of energy $E$ in Figure 1(a). The ratio of $\Gamma_{n}^{W K B}(E)$ to $\Gamma_{n}^{A S}(E)$ is also shown in Figure $1(\mathrm{~b})$. In low-energy region, $\Gamma_{n}^{W K B}$ is larger than $\Gamma_{n}^{A S}$, with a ratio about 4 at $E=0.01 \mathrm{keV}$. The difference becomes smaller as the energy increases. 


\subsection{Resonant cross sections}

With the energy-dependent decay width $\Gamma_{n}(E)$, we achieve the resonant cross section $\sigma_{R}(E)$ from Breit-Wigner formula. In Figure 2, we compare the resonant cross sections $\sigma_{R}(E)$ with a constant width, and those based on two energy-dependent decay widths. It can be clearly seen that the resonance cross sections with a constant width show an abnormal increase in the low-energy region far from the resonance peak. It can be analyzed from From Eq. 2. When $E$ approaches $0 \mathrm{keV},\left(E-E_{r}\right)^{2}+(\Gamma / 2)^{2}$ becomes a constant, $\sigma(E) \propto 1 / E$ in low-energy region. This is completely opposite to the trend of the resonance cross sections evaluated by energy-dependent widths, which becomes smaller as neutron energy decreases. It is more reasonable because lower particle energy away from resonance peak leads to smaller reaction probability, in accordance with lower cross section.

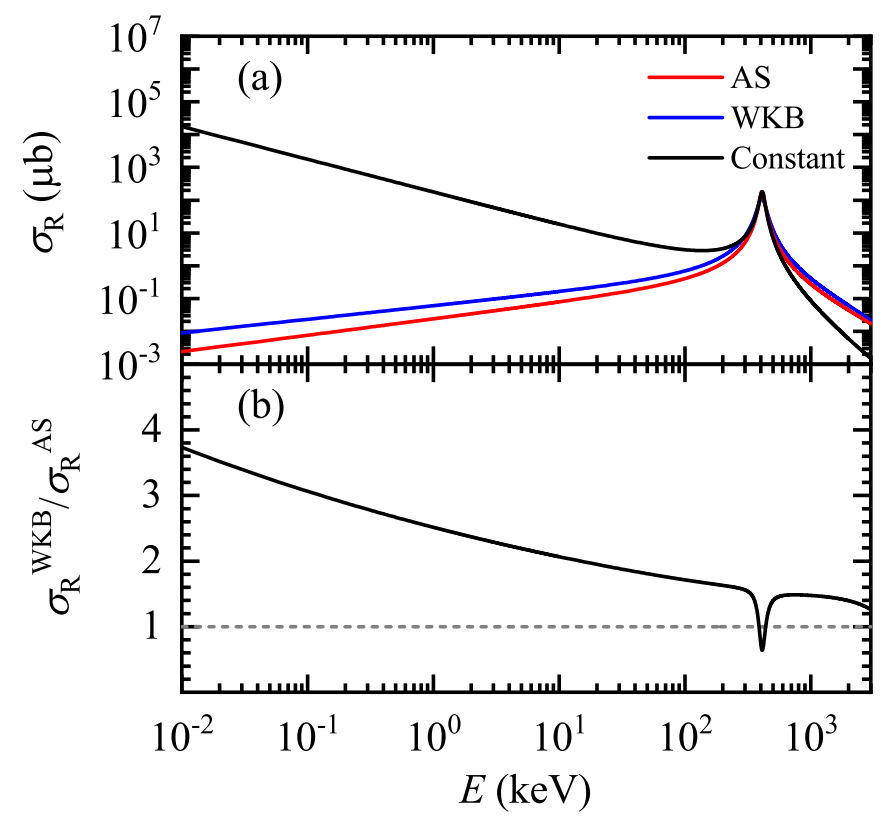

Figure 2. (a) Resonant cross section of $411 \mathrm{keV}$ resonance of ${ }^{16} \mathrm{O}(n, \gamma){ }^{17} \mathrm{O}$, with a constant decay width $\Gamma_{n}=40 \mathrm{keV}$ (black line), and two energy-dependent $\Gamma_{n}(E)$ based the asymptotic solution (red line) and the WKB approximation (blue line). (b) ratio of $\sigma_{R}^{W K B}$ to $\sigma_{R}^{A S}$ as a function of energy $E$.

The ratio of $\sigma_{R}^{W K B}$ to $\sigma_{R}^{A S}$ generally decreases with $E$ increasing, except for the region nearby resonance energy. This can be seen from Eq. (2) that the ratio $\sigma_{R}^{W K B} / \sigma_{R}^{A S}$ is proportional to the ratio $\Gamma_{n}^{W K B} / \Gamma_{n}^{A S}$ when $E$ is far away from resonance energy $E_{r}$. However, the ratio of resonant cross sections rapidly declines around $E_{r}$. This is because the ratio $\sigma_{R}^{W K B} / \sigma_{R}^{A S}$ is proportional to the ratio $\Gamma_{n}^{A S} / \Gamma_{n}^{W K B}$, when $\Gamma_{\gamma} \ll \Gamma_{n}$ and $E \rightarrow E_{r}$. Therefore, compared to $\Gamma_{n}^{A S}$, the larger $\Gamma_{n}^{W K B}$ causes smaller resonant cross section around the resonance energy.

\subsection{Maxwellian-averaged cross sections}

Figure 3 displays the MACSs as a function of $k_{B} T$, based on three decay widths: a constant, $\Gamma_{l}^{W K B}$, and $\Gamma_{l}^{A S}$, respectively. The MACSs with a constant width deviate a lot from the other two, especially for low-energy region, which is consistent with abnormal increase of the 
resonant cross section shown in Figure 2 (a). For energy-dependent widths $\Gamma(E)$, the ratio $\Gamma_{n}^{W K B} / \Gamma_{n}^{A S}$ becomes close to 1.0 (less than $1 \%$ ) when $E>50 \mathrm{keV}$. From this sense, the WKB approximation is also a good way to evaluate MACSs.

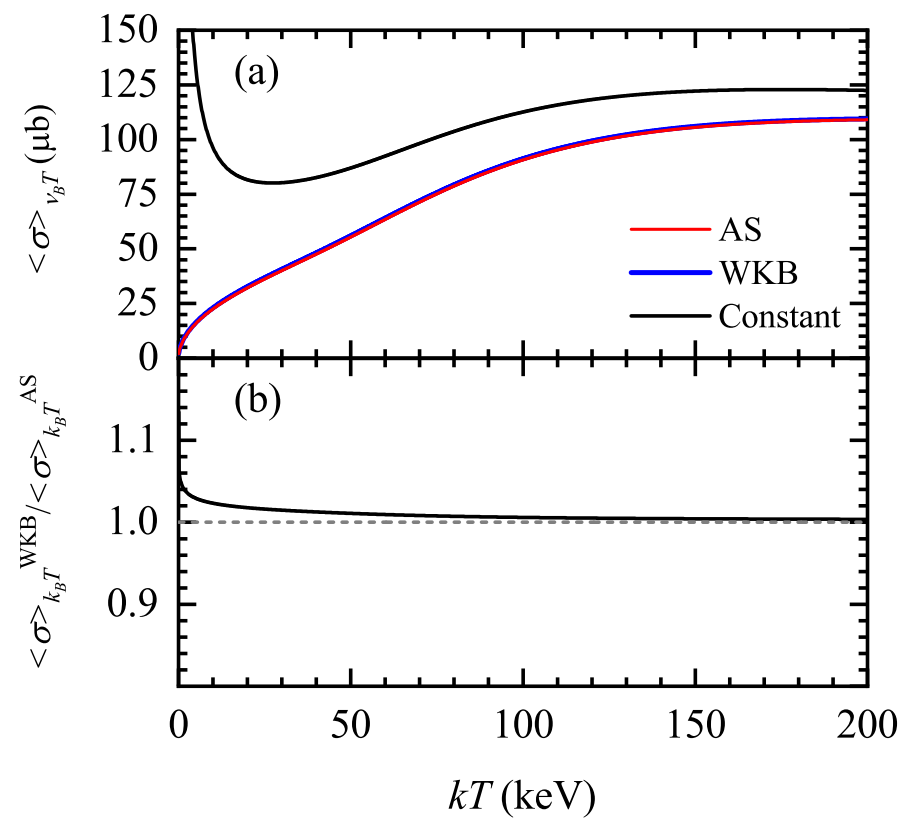

Figure 3. (a) MACSs for ${ }^{16} \mathrm{O}(n, \gamma){ }^{17} \mathrm{O}$ based on three decay widths: constant (black dashed line), $\Gamma_{l}^{W K B}$ (blue line), and $\Gamma_{l}^{A S}$ (red line), respectively. (b) ratio of $\langle\sigma\rangle_{k_{B} T}^{W K B}$ to $\langle\sigma\rangle_{k_{B} T}^{A S}$ as a function of $k_{B} T$.

\section{Summary}

In this paper, we study the impacts of three kinds of decay widths in Breit-Wigner formula on resonant cross sections and MACSs. We found that a constant decay width shows abnormal behavior of the resonant cross sections especially for the low-energy region far away from the resonance peak. Therefore, energy-dependent widths are strongly recommended. Via comparing energy-dependent widths from commonly used WKB approximation and the asymptotic solution, we see that the deviations decrease with the energy goes up. Correspondingly, the ratio of the resonant cross sections shows similar behavior except for the region around the resonance peak. Interestingly, the differences between these two ways shrunk quickly and turn out to be less than $1 \%$ with increasing energy beyond $50 \mathrm{keV}$. Therefore, we make a conclusion that WKB approximation is also a good way to be used for neutron capture process.

\section{References}

[1] P. Mohr, C. Heinz, M. Pignatari, I. Dillmann, A. Mengoni, F. Käppeler, The Astrophysical Journal 827, 29 (2016)

[2] M. He, S.S. Zhang, M. Kusakabe, S. Xu, T. Kajino, The Astrophysical Journal 899, 133 (2020) 
[3] S.S. Zhang, S.Z. Xu, M. He, M.S. Smith, The European Physical Journal A 57, 1 (2021)

[4] C. Bertulani, Computer Physics Communications 156, 123 (2003)

[5] J.J. Sakurai, E.D. Commins, Modern quantum mechanics, revised edition (1995)

[6] M. Caamano, D. Cortina-Gil, W. Mittig, H. Savajols, M. Chartier, C. Demonchy, B. Fernández, M.G. Hornillos, A. Gillibert, B. Jurado et al., Physical review letters 99, 062502 (2007)

[7] S. Ceci, M. Korolija, B. Zauner, Physical review letters 111, 112004 (2013)

[8] I. Hamamoto, Physical Review C 77, 054311 (2008)

[9] D.D. Clayton, Principles of stellar evolution and nucleosynthesis (University of Chicago press, 1983)

[10] A. Trkov, M. Herman, D. Brown et al., Brookhaven National Laboratory 80 (2012)

[11] Y. Nagai, M. Kinoshita, M. Igashira, Y. Nobuhara, H. Makii, K. Mishima, T. Shima, A. Mengoni, Physical Review C 102, 044616 (2020) 